\title{
Empleo de las Espátulas de Velasco como Instrumento de Extracción Fetal
}

\author{
Dr. José Ramb́n López Gómez \\ Trabajo presentado al $X$ Congreso Latinoame- \\ ricano de Obstetricia y Ginecología 1981. \\ Ciudad de Santo Domingo,
}

\section{INTRODUCCION}

El autor en este trabajo realizó un estudio sobre el empleo de un nuevo instrumento obstétrico, ideado por el Dr. Alvaro Velasco, premio Nacional de Medicina, Colombia (1975), el cual ha sido aplicado con regularidad por los médicos que laboramos en el Hospital del Instituto Venezolano de los Seguros Sociales en Puerto Cabello, Servicio de Obstetricia, desde el mes de enero de 1976. Se hizo una revisión retrospectiva de las historias clínicas de pacientes a las cuales se les aplicó las espátulas de Velasco, que así se denomina el instrumento, el cual es una novedosa version de fórceps obstétrico, si así pudiéramos definir, generalizando, el equipo que nos ocupa.

La necesidad de acelerar el trabajo del parto, disminuir las molestias de la madre en el trance del paritorio, corregir las anomalias que puedan obstaculizar el normal tránsito del producto de la concepción en el extremo de salida del

1. DUBROSKY, RICARDO y BRUNO AJEL.LE, FRANCISCO: "Teoría y Práctica del Fórceps". Editorial bibliográfica; Argentina, Buenos Aires 1955. canal del parto, como por ejemplo, una distocia de rotación, una detención del móvil por falta de una adecuada dinámica, etc., ha hecho recurrir a los obstetras de todos los tiempos al uso de aquellos instrumentos capaces de ser como un alargamiento de sus propias manos, para tomar el feto por la porción que se presenta en el canal del parto y poder extraerlo, para beneficio materno o para beneficio de ambos. Así, en el armamentario instrumental del Obstetra hemos visto, desde el punto de vista histórico, desfilar los más diversos y curiosos aparatos, muchos de éllos desprovistos de bases científica para su aplicación y en los que sólo están presentes el ingenio y la acuciosidad del médico o curiosos: "Redes de Seda", "Abrazaderas de barbas de ballena", ganchos, pinzas, instru mentos de mutilación 1 .

Posteriormente aparecen nuevos equipos, con la idea de que al prestar la máxima seguridad, sean también los más inocuos para el binomio materno-fetal: Hawk Denen, Lauff, Kielland, Luickart, Barton, Bamberg, y luego surgen las Espátulas ideadas por Thierry, que abren un nuevo camino dentro del concepto de extracción instrumental del feto y que logran muchos adeptos en sus momentos 
iniciales en que se demuestra su utilidad $y$ ventajas.

En 1975 como ya lo hemos señalado aparece el instrumento ideado por Alvaro Velasco, y el cual viene a ser una reivindicación del antiguo instrumento que ideara Palfyn en 1713.

Los Obstetras hemos deseado siempre disponer de ese Instrumento que garantice la máxima seguridad y el menor daño para la madre y el feto y en esta continua búsqueda nos toca abordar hoy el estudio del instrumento que se presenta.

\section{MODELO TEORICO}

\subsection{El Problema:}

Empleo de las Espátulas de Velasco como instrumento de extracción fetal.

\subsection{Valoración del problema:}

La necesidad que conlleva el estado actual de la práctica médica en el área materno-infantil, a ser cada vez menos agresivos en las prácticas terapéuticas, que puedan aparejar algún grado de traumatismo para el binomio madre-hijo, fundamentalmente en el parto, nos lleva día tras día a la búsqueda de métodos e instrumentos que resultan cada vez más inocuos. Dentro de este orden de ideas y dentro de la concepción del fórceps como instrumento obstétrico útil en la búsqueda de ese diseño ideal, encontramos el instrumento ideado por Alvaro Velasco Chiriboga, que lleva su nombre. Su novedosa concepción, sometida a juiciosos estudios científicos dentro del marco de la composición y acciones de las fuerzas que actuan durante la extracción instrumental del feto, y su facilidad de manejo, le transforman en un recurso de gran utilidad terapéutica y profiláctica durante el segundo período del parto, disminuyendo la morbilidad materno-fetal imputable al trabajo del parto o al uso de los instrumentos ideados para la terminación del mismo.

\subsection{Alcance y limitaciones del estudio:}

En nuestro estudio de carácter retrospectivo, analizando historias clínicas de madres y sus recién nacidos, a quienes se les aplicó las Espátulas de Velasco en el parto, se consideraron los siguientes parámetros. Maternos: edad de la madre, edad de la gestación y paridad, indicación de las Espátulas, duración del trabajo de parto, duración del período expulsivo, tipo de anestesia empleada, y complicaciones maternas atribuibles a la aplicación de las Espátulas (desgarros vagino-perineales $y$ cervicales, edema $y / 0$ hematoma vulvo-perineal). Fetales: variedad de posición fetal y altura de la presentación en el momento de la aplicación de las Espátulas, Apgar del recién nacido al minuto, peso fetal, mortalidad perinatal, condiciones del recién nacido al salir del hospital y lesiones atribuibles a la aplicación de las Espátulas (huellas, equimosis facial, baja puntuación de Apgar, muerte perinatal).

\subsection{Objetivos del estudio:}

1.4.1. Determinar la utilidad de las Espátulas de Velasco, como instrumento de extracción fetal, tomando en consideración los siguientes parámetros:

\section{Atraumaticidad materno-fetal.}

2. Indicación profiláctica y terapéutica.

3. Aplicación por debajo del III Plano de Hodge.

4. Aplicación en diversidad de condiciones en cuanto a edad del embarazo, gestación y paridad. Así como variedad de posición fetal y peso del recién nacido.

1.4.2. Establecer la relación entre las aplicaciones profilácticas $y$ terapéuticas de las Espátulas de Velasco y la puntuación de Apgar de los recién nacidos. 


\subsection{Antecedentes históricos:}

Desde tiempos inmemoriables, el hombre atónito ante el trabajo de parto, se sintió impotente de no poder alargar sus manos, adelgazarlas e introducirlas a través de la vagina y el cuello dilatado, para extraer un feto que estuviera presentandc dificultades para salir al exterior. Es asi como aparecen los primeros intentos $y$ descripciones de fórceps, los modelos de Albucasis y la descripción de Avicenas. Pietre Franco de Lion describe el espéculo de tres ramas "speculum matricis" basado en la experiencia de Rueff (1554), quien presenta un fórceps semejante a una pinza de huesos. Es Peter Chaumberlain quien en 1580 inventa $y$ utiliza el primer instrumento de ramas separables destinadas a la extracción fetal, instrumento que es tenido en el más celoso secreto, siendo un siglo más tarde cuando uno de sus sucesores, Hugo Chamberlain lo hace público acompañado de un profundo fracaso. En 1713 Jean Palfyn, cirujano de Gante crea un instrumento capaz de traccionar sobre la cabeza detenida y el cual consiste en "dos láminas planas de poca superficie, y recurvadas a concavidad interna para adaptarse a la cabeza fetal y prolongadas por dos mangos paralelos" 2 no curvados.

En 1747, aparece en Francia el médico Andre Levret, quien inicia la llamada etapa científica del fórceps y después de ensayar varios modelos adaptados a las circunstancias del canal del parto, introduce la llamada curvatura pelviana de fórceps. Su modelo perfeccionado, tenía una longitud de $42 \mathrm{~cm}$ ! una curvatura pelviana de $6 \mathrm{~cm} /$ de radio, ramas entrecruzadas afrontadas por un sólido tornillo de presión, mangos que terminaban por su extremo libre en un gancho abierto hacia afuera y cucharas fenestra-

2. AURE TULENE, MERCEDES: “Evaluación Fórceps de Hubert-Gilles". Rev. Obst. Ginc. Venezuela, 22:665. 1962 das con amplias ventanas prolongadas hacia el ángulo de la articulación ${ }^{3}$, a fin de poder utilizar lazos que ayudaban a la tracción, anticipándose a la idea de tracción en un eje diferente al de los mangos.

Contemporáneamente con Levret, Smellie en Inglaterra (1752), presenta un instrumento más corto $130 \mathrm{~cm}$ de largo), constituido por dos ramas de articulación cruzada a encaje recíproco y provisto únicamente de curvatura cefálica. Más tarde, Benjamín Pughpresenta su fórceps de ramas articuladas a encaje recíproco, provisto además, de las curvaturas cefálica y pelviana, de una tercera curvatura llamada perineal, a objeto de aminorar las presiones sobre los tejidos blandos maternos. En 1781 Thenance presenta un modelo de fórceps largo de ramas paralelas y divergentes con sistema articular próximo a los ganchos de los mangos. En 1791 aparece el fórceps creado por Busch de ramas cruzadas caracterizado "por ganchos salientes y recurvados próximos a la articulación, para facilitar las maniobras de tracción"4.

En larga sucesión, aparecen nuevos fórceps con hitos marcados por la popularidad del instrumento o por sus aportes en la búsqueda de ese instrumento ideal, que con el menor trauma para la madre y el feto pudiera servir para terminar un parto estancado. Así en el siglo $X I X$, Naegele presenta un fórceps de ramas cruzadas con curvatura cefálica pequeña y pelviana acentuada y juntura mixta por eje y encaje recíproco. También en el siglo $X I X$ aparece el fórceps de Simpson, formado por "dos ramas

3. DUBROSKY, RICARDO Y BRUNO AJE LLO, F.: "Teoría y práctica del Fórceps". Editorial Bibliográfica; Argentina, Buenos Aires, 1955 pp. 22.

4. Ibid. pp. 26. 
cruzadas y articuladas entre sí por un sistema de mortaja de encaje recíproco. Cada rama posee cuchara, porción articular y mangos' 5 . Cada cuchara tiene la curvatura pelviana, doble curvatura cefálica y una espiga que sigue a la cuchara y se acoda en ángulo recto para la articulación del instrumento. "Los mangos son rectos y acanalados para adaptarse mejor a las manos del operador" ${ }^{\prime \prime}$.

En 1877, Tarnier enuncia claramente el principio de la tracción axial y presenta un instrumento de ramas cruzadas, provisto de cucharas fenestradas con doble curvatura pelviana y fetal, zona articular con doble sistema de fijación: tornillo de fijación y tornillo de presión, mangos rectos y paralelos que terminan en ángulos de 90 grados, un sistema de tracción que comienza en los pedículos de las cucharas, y tallos de tracción a los que termina engranándose un tractor provisto de un eje horizontal y otro vertical. Este instrumento se constituye en el ideal para las aplicaciones de fórceps en la excavación (fórceps medio), hoy abandonado.

Más tarde aparece el fórceps de Demelin 8, de ramas paralelas convergentes, donde las cucharas se reunen por arriba y detrás del polo cefálico, convergiendo sus picos en vez de divergir y articulándose sus ramas en la barra de tracción. Nuevos o reactualizados instrumentos aparecen en la escena obstétrica: KieIland, Tucker Mc. Lean, De Lee, Zweifel, Leff, Bartón, Hawks-Dennen, Smith, Hubert-Gilles, Laufe, Luikart, Piper, Bamberg. Diseñados algunos, para sólo resolver problemas muy específicos del parto.

5. URANGA IMAZ, FRANCISCO; URANGA IMAZ (h), F.: "Obstetricia Práctica". 4a. Edición. Intermédica, Editorial Buenos Aires. Argentina, 1977 pp. 837.

6. Ibid.
Siguiendo los principios del paralelismo, Shute idea un fórceps "cuyas ramas se mueven en perfecto paralelismo acomodándose en tal forma a cada cabeza cualquiera sea su talla, las ramas más cortas y más rectas que el fórceps clásico, permiten rotar la cabeza con un ligero movimiento de torsión como el fórceps de Kielland"7. Este fórceps paralelo, puede ser fácilmente aplicable en casi todas las posiciones fetales debido a las características de su curva cefálica y sus ramas angostas.

En 1954 aparecen las Espátulas de Thierry (último modelo), "constituidas por ramas independientes que no se cruzan ni articulan, sus cucharas son anchas sin fenestración con amplia curvatura cefálica y muy moderada curvatura pelviana"8. El contacto con la cabeza fetal se hace a nivel de la región malar y las cucharas deben ser colocadas estrictamente simétricas y paralelas. Thierry hace hincapié en el poco riesgo fetal, por el hecho de que el cráneo queda libre de comprensión; la acción de las mismas sobre los tejidos blandos pelvianos impide la elogación producida por el fórceps $y$ por consiguiente hay menor posibilidad de deslizamiento de las mismas. Monrroy y Colaboradores concluyen que "Ias espátulas son muy benignas para la madre y el feto y muy poco traumáticas para este último"9. Las Espátulas de Thierry y el Retrocéps de Hamon que

7. GREENHILL, J. P.: FRIEDMAN, ENMANUEL A. "Biological Principles and Nodern Practice of Obstetrices". W. B. Saunder Company. Philadelphia. 1979.

8. FEO, CODECIDO ERNESTO: "Principales Métodos Instrumentales de Extracción Fetal". Rev. Obst. Cin. Venezuela. 10:243. 1959.

9. MONROY P., TULIO; CELLY, B.: Col. "Evaluación de las Espátulas de Thierry". Rev. Obst. Gin. Venezuela. 20:439. 1960. 
tomaba la cabeza por detrás, han sido parientes lejanos de las manos de Palfyn, primer intento de extraer el feto con dos palancas no articuladas.

En 1975 Velasco Chiriboga presenta un instrumento basado en los principios de tracción independiente en cada rama, que permita hacer tracción desde la porción facial de la cabeza, eliminando la comprensión craneal. De su instrumento dice Velasco Chiriboga, que fue diseñado "con el deseo de facilitar un instrumento que se ajuste a los preceptos que rigen en la actualidad el auxilio del período expulsivo del parto: fácil aplicación y atraumaticidad"10.

Este Instrumento fue presentado por su autor en el XI Congreso Colombiano de Obstetricia y Ginecología, reunido en Barranquilla, diciembre de 1975. Conjuntamente con una primera "evaluación clínica" hecha por Ramírez Merchán, Velasco Chiriboga y Colaboradores, en la que se señalan las caracter ísticas de ser poco traumáticas, considerándolas "como el instrumento más adecuado, por su inocuidad, para efectos profilácticos y terapéuticos"11. Así como también, se hace mención a que las características de su diseño limitan la altura de su aplicación, con pocas complicaciones y fácil manejo. En ese estudio sobre 756 casos, al revisar morbilidad y mortalidad fetal, no encontramos ningún caso de lesiones derivadas del uso del instrumen-

10. VELASCO CHIRIBOGA, ALVARO.: "Espátulas para la Extracción Fetal un Nuevo Instrumento Obstétrico. Descripción General y Técnica de Aplicación". Rev. Colombiana Obst. y Ginec. XXVI, 1. 1975.

11. RAMIREZ M., RAFAEL; VELASCO CHIRIBOGA, ALVARO y Colaboradores.: "Las Espátulas de Velasco. Evaluación Clínica". Rev. Colombiana Obst. y Ginec. XXVI. 2: 116.1975. to, siendo la patología materna, imputable al uso del mismo, casi insignificante.

El trabajo inédito, "Espátulas vs. Forceps", de Beckman y Barreto (Bogotá), los autores encontraron que "las lesiones maternas fueron más frecuentes e importantes en las pacientes a las cuales se aplicaron fórceps tanto en forma global como proporcionalmente, de acuerdo a la maniobra realizada" 12 . $E$ igualmente señalan que "la morbilidad fetal, fue mucha más marcada con los fórceps que con las espátulas"13. García Gamboa en su trabajo inédito "Las Espátulas de Velasco", (Puerto Rico), considera a este "Novel Instrumento de Velasco, como excelente, útil y poco traumático al binomio feto-materno"14. Entre nosotros, las Espátulas de Velasco se vienen utilizando desde enero de 1976, Ilevándonos a realizar el presente trabajo de evaluación del uso de las mismas.

\subsection{Definición de términos:}

1.6.1. Espátulas de Velasco: instrumento de extracción fetal, constituido por dos ramas paralelas, no fenestradas y no articuladas, de $25 \mathrm{~cm}$ de longitud y $3.5 \mathrm{~mm}$. de espesor. Su porción más amplia tiene $5 \mathrm{~cm}$ ide ancho y la porción intermedia $2 \mathrm{~cm}$ ، y está terminada por una porción denominada mango que la cruza transversalmente $y$ cuya longitud es de $5 \mathrm{~cm}$.

12. BECKMAN S., ENRIQUE; BARRETO S. FRANCISCO: "Espátulas vs. Fórceps". Trabajo inédito. Universidad del Rosario. Facultad de Medicina. Bogotá, Colombia. 1975, p. 15.

13. Ibid.

14. GARCIA G., MARIO: “Las Espátulas de Velasco". Trabajo inédito. Universidad de Puerto Rico. Recinto de Ciencias Médicas. Escuela de Medicina. Puerto Rico. 1975. 25. 
1.6.2. Extracción fetal instrumental: consiste en la aplicación de las Espátulas de Velasco sobre el polo cefálico fetal, la rotación del mismo cuando sea necesario y la tracción en el sentido de las fuerzas expulsivas, siguiendo el eje del canal del parto.

1.6.3. Utilidad de la Espátula de Velasco: factibilidad de su empleo como instrumento de extracción fetal, tomando en consideración los siguientes parámetros:

1. Atraumaticidad materno-fetal.

2. Indicación profiláctica y terapéutica.

3. Aplicación por debajo del III Plano de Hodge.

4. Aplicación bajo analgesia, sedación o anestesia local y troncular.

5. Aplicación en diversidad de condiciones en cuanto a edad del embarazo, gestación y paridad, así como variedad de posición fetal y peso del recién nacido.

1.6.4. Atraumaticidad materno-fetal: capacidad de las Espátulas de Velasco, de no producir o producir el mínimo. Lesiones maternas que pudieran atribuirse a su aplicación: desgarros vaginoperineales, cervicales, edema y/o hematomas vulvo-perineales; en el recién nacido: huellas de las espátulas, equimosis facial, convulsiones, baja puntuación apgar, muerte peri-natal.

1.6.5. Indicación profiláctica: Basados en el concepto introducido por De Lee en 1920, se consideran como espátulas indicadas profilácticamente, aquéllas que se aplicaron en el IV Plano de Hodge, con la finalidad de acortar el período expulsivo para reducir el esfuerzo y las molestias maternas, evitar la sobre distensión de los tejidos blandos y preservar el cerebro fetal de la comprensión prolongada contra el periné.
1.6.6. Indicación terapéutica: son aquellas en que la aplicación de las espátulas se hizo por existir alguna patología propia del parto, (expulsivo prolongado. rotación detenida), sufrimiento fetal $u$ otra patología materna que pueda ser agravada por la prolongación del período expulsivo.

\subsubsection{III y IV Plano de Hodge:} los Planos de Hodge, son coordenadas obstétricas que permiten precisar la altura en que se encuentra la cabeza fetal en un momento determinado del parto. EI III Plano, pasa a nivel de las espinas ciáticas y corresponden a la estación 0 de la calsificación de De Lee. El intermedio entre III y IV Planos de Hodge, coresponde a las estaciones $(+1,+2 y$ +3 ) de De Lee; y el IV Plano, paralelo al anterior, pasa a nivel de la punta del coxis no retropulsado $y$ "comprende las estaciones $(+4 y+5)$ de De Lee"15.

\section{MODELO OPERACIONAL}

\subsection{Método:}

Se realizó un estudio explorativo sobre el empleo de las Espátulas de Velasco como instrumento de extracción fetal.

\subsection{Población de interés:}

Se tomó como población de interés 399 historias de parturientas y las de sus respectivos recién nacidos, a quienes se les aplicó las Espátulas de Velasco en el segundo período del parto, en el Servicio de Obstetricia del Instituto Venezolano de los Seguros Sociales en Puerto CabeIlo, durante el período de 1976-1980.

\subsection{Muestra:}

De las 399 aplicaciones de Espátulas de Velasco, registradas en los Libros de

15. HUGHES, EDWARD: "Terminonogía en Obstetricia y Ginecología". Salvat Editores. Barcelona. España. 1975. p. 428. 
Cuadro No. 1

PARTOS SEGUN AÑO Y FORMA DE TERMINACION DEL PARTO. EXPRESADO EN \% HOSPITAL DEL I.V.S.S.* PUERTO CABELLO, VENEZUELA QUINQUENIO 1976-1980

\begin{tabular}{|c|c|c|c|c|c|c|c|c|c|c|}
\hline & \multicolumn{8}{|c|}{ FORMAS DE TERMINACION DEL PARTO } & \multirow{3}{*}{\multicolumn{2}{|c|}{$\begin{array}{c}\text { Total de \% } \\
\text { Partos }\end{array}$}} \\
\hline & \multirow{2}{*}{\multicolumn{2}{|c|}{$\begin{array}{c}\text { Espontáneo } \\
\text { Frecuencia } \%\end{array}$}} & \multirow{2}{*}{\multicolumn{2}{|c|}{$\frac{\text { Cesárea }}{\text { Frecuencia \% }}$}} & \multirow{2}{*}{\multicolumn{2}{|c|}{$\begin{array}{c}\text { Espátulas de Velasco } \\
\text { Frecuencia } \%\end{array}$}} & \multicolumn{2}{|c|}{ Fórceps } & & \\
\hline & & & & & & & Fre & cia \% & & \\
\hline 1976 & 1.782 & 88.74 & 148 & 6.37 & 45 & 2.24 & 33 & 1,64 & 2.008 & 100 \\
\hline 1977 & 1.727 & 84.99 & 210 & 10.33 & 25 & 4.18 & 10 & 0.49 & 2.032 & 100 \\
\hline 1978 & 1.746 & 84.72 & 223 & 10.82 & 75 & 3.64 & 17 & 0.82 & 2.061 & 100 \\
\hline 1979 & 1.759 & 80.72 & 271 & 12.44 & 126 & 5.78 & 23 & 1.05 & 2.179 & 100 \\
\hline 1980 & 1.458 & 81.91 & 81.91 & 11.55 & 68 & 3.79 & 49 & 2.73 & 1.792 & 100 \\
\hline TOTAL & 8.482 & 84.21 & 1.050 & 10.51 & 399 & 3.96 & 132 & 1.31 & 10.072 & 100 \\
\hline
\end{tabular}

Fuente: Archivo de Historias Médicas del Hospital del * Instituto Venezolano de los Seguros Sociales en Puerto Cabello

Nacimientos del Servicio de Obstetricia del Instituto Venezolano de los Seguros Sociales en Puerto Cabello, en el período 1976-1980, solamente 261 fueron incluidos como muestra, por el hecho de que 88 historias, no ten ían todos los datos del recién nacido, requeridos para este estudio y 50 historias maternas, no aparecieron en el archivo.

\section{RESULTADOS}

La distribución total de partos durante el quinquenio 1976-1980, según su forma de terminación se muestra en el Cuadro No. 1.

En él podemos observar que de un total de 10.072 partos, $10.51 \%$ terminaron por cesárea y $5.27 \%$ fueron partos instrumentales, de los cuales $3.96 \%$ correspondieron a la aplicación de Espátulas de Velasco y $1.31 \%$ a la aplicación de otros fórceps. La incidencia de aplicación de las espátulas varió entre el $2.24 \%$ para el primer año de estudio (1976), y el $5.78 \%$ para el año de 1979.

En el Gráfico Unico se muestra una línea de tendencia ligeramente ascendente en la aplicación de las Espátulas, con un aumento promedio de $1.1 \%$.

En el cuadro No. 2 podemos observar que la mayor probabilidad de aplicación de espátulas correspondió a las primíparas $53.16 \%$ y a las grandes multíparas $16.09 \%$. Estas probabilidades varíanentre el $10.70 \%$ para el grupo de las VI y VIII Paras, $8.74 \%$ para el grupo de las II y III paras, y $8.66 \%$ para el grupo de las IV y $\mathrm{V}$ paras.

En el cuadro No. 3 observamos una mayor probabilidad de aplicación de las espátulas $40.04 \%$ en parturientas con 
edades comprendidas entre los 15 y 19 años, en segundo lugar $28.54 \%$ en el grupo comprendido entre 20 y 24 años y en tercer lugar $23.14 \%$ en el grupo de 40 a 44 años.

Cuadro No. 2

APLICACIONES DE ESPATULAS DE VELASCO SEGUN PARIDAD. EXPRESADO EN TASAS POR MIL

HOSPITAL DEL I.V.S.S * PUERTO CABELLO, VENEZUELA QUINOUENIO 1976.1980

\begin{tabular}{|lccc|}
\hline Paridad & Total de Partos & $\begin{array}{c}\text { Aplicaciones de } \\
\text { Espátulas de Velasco }\end{array}$ & Tasa por mis \\
\hline Primíparas & 3.649 & 194 & 53.16 \\
II - III & 2.403 & 21 & 8.74 \\
IV - V & 1.155 & 10 & 8.66 \\
VI - VII & 1.028 & 11 & 10.70 \\
VIII y Más & 1.367 & 22 & 16.09 \\
No precisada & 470 & 3 & - \\
\hline TOTAL & 10.072 & 261 & 26.0 \\
\hline
\end{tabular}

Fuente: Archivo de Historias Médicas del Hospital del "Instituto Venezolano de I Seguros Sociales en Puerto Cabello.

En el cuadro No. 4 observamos que la tasa más alta de probabilidad de aplicación de las espátulas $34.37 \%$ correspondió a gestaciones sobre el término, siguiéndole en probabilidad el $30.54 \%$ para aquellos embarazos cronólogicamente prolongados, $29.98 \%$ para embarazos entre 38 y 39 serhanas y el $24.97 \%$ para los embarazos entre las 32 y 33 semanas.

GRAFICO UNICO

APLICACIONES DE ESPATULAS DE VELASCO, EXPRESADAS EN \% Y PROMEDIO HOSPITAL DEL I.V.S.S. PUERTO CABELLO, VENEZUELA. QUINOUENIO 1976-1980

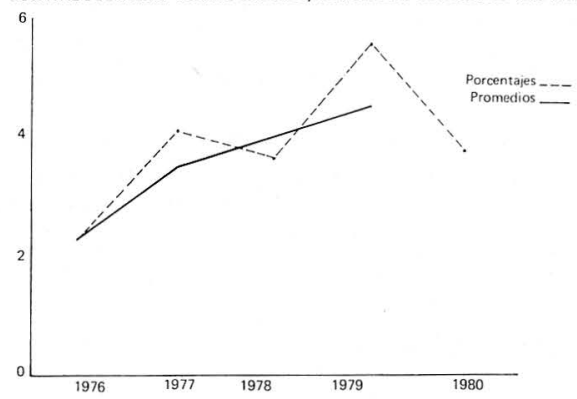

Cuadro No. 3

APLICACIONES DE ESPATULAS DE VELASCO SEGUN EDAD DE LAS PARTURIENTAS, EXPRESADO EN TASA POR MIL

HOSPITAL DEL I.V.S.S. PUERTO CABELLO, VENEZUELA QUINQUENIO 1976-1980

\begin{tabular}{|cccc|}
\hline EDAD & Total de Partos & $\begin{array}{c}\text { Aplicaciones de } \\
\text { Espátulas de Velasco }\end{array}$ & Tasa por mil \\
\hline $15-19$ & 1.798 & 72 & 40.04 \\
$20-24$ & 3.538 & 101 & 28.54 \\
$25-29$ & 2.736 & -51 & 18.64 \\
$30-34$ & 990 & 16 & 16.16 \\
$35-39$ & 689 & 14 & 20.31 \\
$40-44$ & 216 & 5 & 23.14 \\
No precisado & 105 & 2 & - \\
\hline TOTAL & 10.072 & 261 & 26.0 \\
\hline
\end{tabular}

Fuente: Archivo de Historias Médicas del Hos. pital del *Instituto Venezolano de los Seguros Sociales en Puerto Cabello.

El cuadro No. 5 nos muestra un aumento progresivo en la probabilidad de aplicación de las espátulas $10.95 \%$ a $74.02 \%$ a medida que aumenta la duración del trabajo de parto, notándose un incremento marcado en aquellos trabajos de partos con una duración igual o mayor de 12 horas.

Cuadro No. 4

APLICACIONES DE ESPATULAS DE VELASCO SEGUN EDAD GESTACIONAL EXPRESADO EN TASAS POR MIL HOSPITAL DEL I.V.S.S.* PUERTO CABELLO, VENEZUELA QUINQUENIO 1976-1980

\begin{tabular}{|cccc|}
\hline $\begin{array}{c}\text { Edad Gestacional } \\
\text { (en semanas) }\end{array}$ & Total de partos & $\begin{array}{c}\text { Aplicaciones de } \\
\text { Espátulas de Velasco }\end{array}$ & Tasa por mil \\
\hline $32-33$ & 1.001 & 25 & 24.97 \\
$34-35$ & 591 & 6 & 10.5 \\
$36-37$ & 1.709 & 25 & 14.62 \\
$38-39$ & 2.768 & 83 & 29.98 \\
$40-41$ & 3.200 & 97 & 34.37 \\
$42-43$ & 622 & 19 & 30.54 \\
No precisada & 181 & 6 & - \\
\hline TOTAL & 10.072 & 261 & 26.0 \\
\hline
\end{tabular}


Cuadro No. 5

\section{APLICACIONES DE ESPATULAS DE VELASCO SEGUN DURACION DE PARTOS EN HORAS, EXPRESADO EN TASAS POR MIL}

HOSPITAL DEL I.V.S.S.* PUERTO

CABELLO, VENEZUELA

QUINQUENIO 1976-1980

\begin{tabular}{|crcc|}
\hline $\begin{array}{c}\text { Duración del } \\
\text { trabajo de } \\
\text { parto (en horas) }\end{array}$ & $\begin{array}{c}\text { Total de } \\
\text { Partos }\end{array}$ & $\begin{array}{c}\text { Aplicaciones } \\
\text { de Espátulas } \\
\text { de Velasco }\end{array}$ & Tasa por mil \\
\hline $0-5$ & 2.555 & 28 & 10.95 \\
$6-11$ & 4.243 & 101 & 23.80 \\
$12-17$ & 1.855 & 90 & 48.51 \\
$18-23$ & 560 & 29 & 51.78 \\
$24-29$ & 142 & 8 & 56.33 \\
$30-35$ & 47 & 3 & 63.82 \\
$36-41$ & 27 & 2 & 74.02 \\
No precisadas & 643 & - & - \\
\hline TOTAL & 10.072 & 261 & 26.0 \\
\hline
\end{tabular}

Fuente: Archivo de Historias Médicas del Hospital del * Instituto Venezolano de los Seguros Sociales en Puerto Cabello.

Cuadro No. 6: observamos que la probabilidad de aplicación de las espátulas, aumenta progresivamente de $14.94 \%$ a $32.02 \%$ en la medida que aumenta la duración del período expulsivo de menos de 30 minutos hasta 90 y más minutos. Notándose $32.02 \%$ de probabilidades de aplicación para aquellos casos en los que el período expulsivo sobrepasó los 90 minutos: $29.37 \%$ para los períodos expulsivos comprendidos entre los 60 y 89 minutos y $18.26 \%$ para los períodos expulsivos comprendidos entre los 30 y los 59 minutos.
En el cuadro No. 7 notamos que el mayor número de aplicaciones de espátulas $51.50 \%$ fueron aplicadas entre el III y IV Plano de Hodge y el $48.50 \%$ en el IV Plano.

Cuadro No. 6

\section{APLICACIONES DE ESPATULAS DE VELASCO SEGUN DURACION DEL PERIODO EXPULSIVO \\ (EN MINUTOS), EXPRESADO EN TASAS POR MIL}

HOSPITAL DEL I.V.S.S. PUERTO CABELLO VENEZUELA QUINQUENIO 1970-1980

\begin{tabular}{|cccc|}
\hline $\begin{array}{c}\text { Duración de } \\
\text { Período } \\
\text { expulsivo } \\
\text { (en minutos) }\end{array}$ & $\begin{array}{c}\text { Total de } \\
\text { partos }\end{array}$ & $\begin{array}{c}\text { Aplicaciones } \\
\text { de Espátulas } \\
\text { de Velasco }\end{array}$ & Tasa por mil \\
\hline $\begin{array}{c}\text { Menos de } 30 \\
\text { minutos } \\
30-59\end{array}$ & 2.945 & 44 & 14.94 \\
$60-89$ & 2.860 & 84 & 29.37 \\
$90-119$ & 687 & 22 & 37.02 \\
No precisado & 349 & 52 & \\
\hline TOTAL & 10.072 & 261 & 26 \\
\hline
\end{tabular}

Fuente: Archivo de Historias Médicas del Hospital del Instituto Venezolano de los Seguros Sociales en Puerto Cabello.

Cuadro No. 8: observamos que la mayor frecuencia de aplicación, de acuerdo a la variedad de posición fetal, ocurrió en las variedades anteriores occipito púbicas $88.42 \%$. En occipito sacra, $8.49 \%$ en derecha transversa $2.70 \%$ y en izquierda transversa $0.39 \%$.

Cuadro No. 9: de los 261 casos estudiados el $67.04 \%$ de las aplicaciones del 
Cuadro No. 7

\section{APLICACIONES DE ESPATULAS DE VELASCO SEGUN ALTURA DE LA PRESENTACION (EN PLANOS DE HODGE). EXPRESADO EN $\%$ HOSPITAL DEL I.V.S.S. PUERTO CABELLO, VENEZUELA QUINOUENIO 1976-1980}

\begin{tabular}{|crc|}
\hline $\begin{array}{c}\text { Altura de la presentación } \\
\text { (en planos de Hodge) }\end{array}$ & Frecuencia & $\%$ \\
\hline III - IV & 120 & 51.50 \\
IV & 113 & 48.50 \\
No precisada & 28 & - \\
\hline TOTAL & 261 & 100. \\
\hline
\end{tabular}

Fuente: Archivo de Historias Médicas del Hospital del *Instituto Venezolano de los Seguros Sociales en Puerto Cabello.

Espacio intermedio entre III y IV Plano de Hodge que corresponde a las estaciones +2 y +3 de De Lee.

instrumento fueron por indicación terapéutica y el $32.95 \%$ profilácticas.

En el cuadro No. 10 se desglosan las indicaciones terápeuticas de las espátulas; observamos la mayor frecuencia $56 \%$ para los casos de período expulsivo prolongado, siguiéndole $16 \%$ para la combinación distocia de rotación, con trabajo de parto prolongado y expulsivo prolongado, y en cifras sucesivas, $9.14 \%$ para el trabajo de parto prolongado $y$ $5.71 \%$ para el trabajo de parto $\mathrm{V}$ expulsivo prolongados con sufrimiento fetal. Con el mismo rango $5.71 \%$ se señalan los casos de cesáreas anteriores, con $4 \%$ la pre-eclampsia y con $3.43 \%$ el sufrimiento fetal.

Cuadro No. 11: muestra que el mayor porcentaje $60.15 \%$, de aplicaciones del instrumento se hizo con anestesia puden- da bilateral. En el $37.16 \%$ se empleo anestesia local infiltrativa y sólo en el $2.68 \%$ se utilizó anestesia peridural.

Cuadro No. 12: en el $90.80 \%$ de los casos estudiados no hubo morbilidad y que las lesiones maternas habidas se refieren a desgarros cervicales $5.36 \%$ y desgarros perineales $3.83 \%$.

Cuadro No. 13: la mayor probabilidad de aplicación de las espátulas de $29.58 \%$ o a $30.61 \%$ ocurrió en recién nacidos con peso menor de 2.500 gramos y sigue en probabilidad de aplicación $27.33 \%$ en recién nacidos con pesos comprendidos entre los 4.000 y los 4.499 gramos correspondiendo la menor probabilidad de aplicaciones a recién nacidos entre 3.000 y 3.499 gramos.

Cuadro No. 14: muestra la frecuencia de aplicación de las espátulas por unidad

Cuadro No. 8

APLICACIONES DE ESPATULAS DE VELASCO SEGUN VARIEDAD DE POSICION FETAL. EXPRESADO EN $\%$ HOSPITAL DEL I.V.S.S.* PUERTO CABELLO, VENEZUELA QUINQUENIO 1976-1980

\begin{tabular}{|lrc|}
\hline Variedad de Posición & Frecuencia & \multicolumn{1}{c|}{$\%$} \\
\hline Occipito Púbica & 229 & 88.42 \\
Occipito Sacra & 22 & 8.49 \\
Derecha Transversa & 7 & 2.70 \\
Izquierda Transversa & 1 & 0.39 \\
No Registrada & 2 & - \\
\hline TOTAL & 261 & 100. \\
\hline
\end{tabular}

Fuente: Archivo de Historias Médicas del Hospital del ${ }^{*}$ Instituto Venezolano de los Seguros Sociales en Puerto Cabello. 
Cuadro No. 9

APLICACIONES DE

ESPATULAS DE VELASCO SEGUN

SU INDICACION PROFILACTICA Y TERAPEUTICA, EXPRESADO EN $\%$

HOSPITAL DEL I.V.S.S.* PUERTO CABELLO, VENEZUELA QUINQUENIO 1976-1980

\begin{tabular}{|lcc|}
\hline Indicación & Frecuencia & $\%$ \\
\hline Terapéutica & 175 & 67.04 \\
Profiláctica & 86 & 32.95 \\
\hline TOTAL & 261 & 100. \\
\hline
\end{tabular}

Fuente: Archivo de Historias Médicas del Hospital del *Instituto Venezolano de los Seguros Sociales en Puerto Cabello.

Cuadro No. 10

\section{APLICACIONES TERAPEUTICAS DE} ESPATULAS DE VELASCO SEGUN CAUSA DE INDICACION DE LAS MISMAS, EXPRESADO EN \%

HOSPITAL DEL I.V.S.S.*

PUERTO CABELLO, VENEZUELA QUINQUENIO 1976-1980

\begin{tabular}{|ccc|}
\hline $\begin{array}{c}\text { Causa de indicación de las } \\
\text { espátulas }\end{array}$ & Frecuencia & $\%$ \\
\hline $\begin{array}{c}\text { Expulsivo prolonqado } \\
\text { Distocia de rotación con trabajo de } \\
\text { parto prolongado y expulsivo prolongado } \\
\text { Trabajo de parto prolongado }\end{array}$ & 98 & 56. \\
$\begin{array}{c}\text { Trabajo de parto prolongado y expulsivo } \\
\text { prolongado con sufrimiento fetal }\end{array}$ & 16 & 16. \\
Césarea anterior & 10 & 5.14 \\
Pre-Ecampsia y Eclampsia & 10 & 5.71 \\
Sufrimiento fetal & 7 & 4. \\
TOTAL & 6 & 3.43 \\
\hline
\end{tabular}

Fuente: Archıvo de Historias Médicas del Hospital del ${ }^{*}$ Instituto Venezolano de los Seguros Sociales en Puerto Cabello.
Cuadro No. 11

APLICACIONES DE ESPATULAS DE VELASCO SEGUN TIPO DE ANESTESIA UTILIZADA, EXPRESADA EN \%

HOSPITAL DEL I.V.S.S.* PUERTO CABELLO, VENEZUELA QUINQUENIO 1976-1980

\begin{tabular}{|lcc|}
\hline Tipo de Anestesia & Frecuencia & $\%$ \\
\hline Pudenda bilateral & 157 & 60.15 \\
Local & 97 & 37.16 \\
Peridural & 7 & 2.68 \\
\hline TOTAL & 261 & 100. \\
\hline
\end{tabular}

Fuente: Archivo de Historias Médicas del Hospital del ${ }^{*}$ Instituto Venezolano de los Seguros Sociales en Puerto Cabello.

Cuadro No. 12

APLICACIONES DE ESPATULAS DE VELASCO SEGUN LESIONES MATERNAS ATRIBUIBLES A LAS MISMAS, EXPRESADO EN \% HOSPITAL DEL I.V.S.S.* PUERTO CABELLO, VENEZUELA QUINQUENIO 1976-1980

\begin{tabular}{|lcr|}
\hline Lesiones maternas & Frecuencia & \multicolumn{1}{c|}{$\%$} \\
\hline Ninguna & 237 & 90.80 \\
Desgarro cervical & 14 & 5.36 \\
Desgarro perineal & 10 & 3.83 \\
\hline TOTAL & 261 & 100. \\
\hline
\end{tabular}

Fuente: Archivo de Historias Médicas del Hospital del *Instituto Venezolano de los Seguros Sociales en Puerto Cabello. 
de puntuación de Apgar de los recién nacidos estudiadas, notándose en el grupo de recién nacidos, cuyo Apgar fue de 7 a 10 puntos, una frecuencia por unidad de 57.5 mientras que en el grupo conApgar de 0 a 3 puntos la frecuencia por unidad fue de 1.

El cuadro No. 15 muestra que en los 261 casos estudiados, de 86 a quienes se aplicó profilácticamente, $96.5 \%$ tuvieron Apgar igual o por encima de siete (7) puntos y sólo 3.48 presentaron una puntuación inferior a siete (7).

La prueba Chi cuadrado demostró diferencias estadísticamente significativas $(P<0.05)$ entre la puntuación de Apgar en ambos grupos lo cual nos lleva a aceptar que las probabilidades de obtener un neonato con puntuación de Apgar igual o mayor de siete (7) son significativamente altas cuando la aplicación de las espátulas se hace filácticamente.

Cuadro No. 16: en el 94.25 de los recién nacidos no hubo ninguna lesión atribuibles a la aplicación del instrumento Sólo se señalan, huella facial unilateral en el $2.30 \%$ y hematoma parietal izquierdo $1.15 \%$. El resto de lesiones descritas (escoriación región frontal derecha, escoriación región cilar derecha, edema región parietal derecha, edema occipito parietal, hematoma biparietal y parálisis facial) tienen cada una de ellas una incidencia de $0.38 \%$, lo que sumado representa una morbilidad global de $5.73 \%$.

\section{COMENTARIOS}

Señalamos la mayor probabilidad de aplicación de las espátulas en las primíparas, lo cual es coincidente con lo expresado por Ramírez Merchán y Col (20); pero además hacemos notar que en nuestra casuística existe una alta
Cuadro No. 13

APLICACIONES DE ESPATULAS DE VELASCO SEGUN PESO

DE LOS RECIEN NACIDOS

(EN GRAMOS), EXPRESADO EN TASAS POR MIL

\section{HOSPITAL DEL I.V.S.S.* \\ PUERTO CABELLO. VENEZUELA QUINQUENIO 1976-1980}

\begin{tabular}{|cccc|}
\hline $\begin{array}{c}\text { Peso de los recién } \\
\text { nacidos (en gramos) }\end{array}$ & $\begin{array}{c}\text { Total de } \\
\text { nacimientos }\end{array}$ & $\begin{array}{c}\text { Aplicaciones de } \\
\text { Espátulas de Velasco }\end{array}$ & Tasa por mil \\
\hline $1.500-1.999$ & 98 & 3 & 30.61 \\
$2.000-2.499$ & 1.555 & 46 & 29.58 \\
$2.500-2.999$ & 1.763 & 48 & 27.22 \\
$3.000-3.499$ & 4.973 & 118 & 23.72 \\
$3.500-3.999$ & 1.008 & 27 & 26.78 \\
$4.000-4.499$ & 695 & 19 & 27.33 \\
\hline TOTAL & 10.092 & 261 & 26.0 \\
\hline
\end{tabular}

Fuente: Archivo de Historias Médicas del Hospital del *Instituto Venezolano de los Seguros Sociales en Puerto Cabello.

Cuadro No. 14

APLICACIONES DE ESPATULAS DE VELASCO SEGUN APGAR DE LOS RECIEN NACIDOS.

EXPRESADO EN FRECUENCIA POR UNIDAD.

HOSPITAL DEL I.V.S.S.* PUERTO

CABELLO. VENEZUELA

QUINQUENIO 1876-1980

\begin{tabular}{|ccc|}
\hline Puntuación de Apgar & Frecuencia & $\begin{array}{c}\text { Frecuencia por puntuación } \\
\text { de Apgar }\end{array}$ \\
\hline $0-3$ & 3 & 1.0 \\
$4-6$ & 28 & 9.3 \\
$7-10$ & 230 & 57.5 \\
\hline TOTAL & 261 & - \\
\hline
\end{tabular}

Fuente: Archivo de Historias Médicas del Hospital del * Instituto Venezolano de los Seguros Sociales en Puerto Cabello. 
Cuadro No. 15

\section{RELACION ENTRE APLICACIONES PROFILACTICAS $Y$ TERAPEUTICAS DE ESPATULAS DE VELASCO Y APGAR DE LOS RECIEN NACIDOS. EXPRESADO EN \%}

\section{HOSPITAL DEL I.V.S.S.* PUERTO CABELLO, VENEZUELA QUINQUENIO 1976-1980}

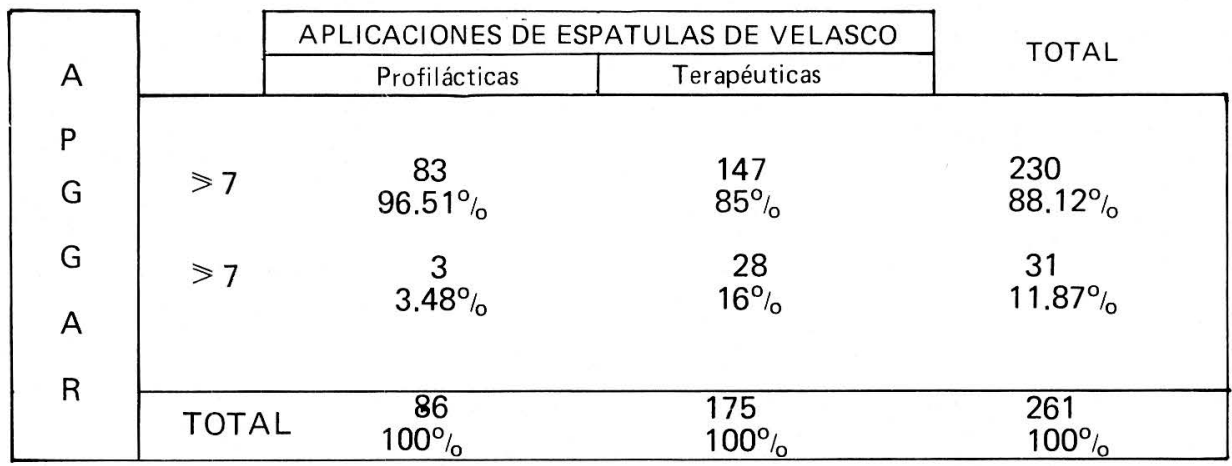

Fuente: Archivo de Historias Médicas del Hospital del * Instituto Venezolano de los Seguros Sociales en Puerto Cabello.

incidencia de aplicaciones grandes multíparas, lo que pone en evidencia que este grupo de parturientas también requiere una ayuda más efectiva durante el segundo período del parto.

En cuanto a la edad de las parturientas, la mayor probabilidad de aplicación estuvo comprendida entre 15 a 24 años, hallazgos que son semejantes a los mencionados por Ramírez Merchán y Col (20); la explicación estaría en relación con el mayor número de partos que ocurren, entre estas edades de la vida.

Las elevadas tasas de aplicaciones en embarazos cronológicamente prolongados y en embarazos menores de 34 semanas, evidencias que la post-madurez y lapre-madurez constituyeron factores a considerar en la aplicación del instrumento. Estos hallazgos, difieren de lo reportado por Beckmann y Barreto (4).
El período expulsivo prolongado, como primera indicación de aplicación terapéutica de las Espátulas de Velasco en este estudio, es otro aspecto coincidente con lo señalado por Ramírez Merchán y Col. (20).

El hecho de que todas las aplicaciones se realizaron por debajo del III Plano de Hodge, con los beneficios que esto conlleva para el producto de la concepción sometido a un parto instrumental, responde a uno de los objetivos de inventor de las espátulas, de lograr un instrumento, que por sus características, evite las tomas altas. Este mismo hecho de la aplicación del instrumento en los planos más bajos de la pelvis explica la razón por la cual, la mayoría de nuestros casos fueron resueltos con anestesia pudenda bilateral y anestesia local infiltrativa.

El mayor porcentaje de aplicación de las espátulas, por indicación terapéutica, 
Cuadro No. 16

\section{APLICACIONES DE ESPATULAS DE VELASCO SEGUN \\ LESIONES FETALES ATRIBUIBLES A LAS MISMAS. EXPRESADO EN $\%$}

HOSPITAL DEL I.V.S.S.* PUERTO CABELLO, VENEZUELA QUINQUENIO 1976-1980

\begin{tabular}{|ccr|}
\hline Lesiones fetites & Frecuencia & $\%$ \\
\hline Ninguna & 246 & 94.25 \\
Huella facıal unilateral & 6 & 2.30 \\
Hematoma parietal izquierdo & 3 & 1.15 \\
Escoriación región frontal derecha & 1 & 0.38 \\
Escoriación región ciliar derecha & 1 & 0.38 \\
Edema parietal derecho & 1 & 0.38 \\
Edema occipito parietal & 1 & 0.38 \\
Hematoma biparietal & 1 & 0.38 \\
Parálisis facial & 1 & 0.38 \\
\hline TOTAL & 261 & 100. \\
\hline
\end{tabular}

Fuente: Archivo de Historias Médicas del Hospital del * Instituto Venezolano de los Seguros Sociales en Puerto Cabello.

evidencia que en nuestro medio, el instrumento ideado por Velasco, ha sido aceptado, más como recurso capaz de resolver distocias intraparto que como elemento de utilidad profiláctica, como lo reportan Ramírez Merchán y Col (20).

Nuestras cifras porcentuales de $9.19 \%$ de lesiones maternas atribuibles al uso de las espátulas son algo mayores que las reportadas por Ramírez Merchan y Col (20), pero consideramos importante señalar que la mayoría de los desgarros perineales se describen como una prolongación de la episiotomía y que el $95 \%$ de los desgarros cervicales reportados, corresponden a un mismo operador, en el año en que se comenzó a utilizarlas, hallazgos éstos que nos hacen presumir que las lesiones maternas reportadas deben ser atribuibles más a la falta de pericia del operador que al instrumento mismo.

El hecho de que el mayor porcentaje $88.12 \%$ de los recién nacidos tuvieran un Apgar igual o mayor de 7 y que de los recién nacidos con Apgar por debajo de 3 , uno fue un mortinato con diagnóstico previo de muerte fetal in útero $y$ otro fue un desnutrido in útero con sufrimiento fetal agudo, que falleció al nacer, pone en evidencia la inocuidad del instrumento para el feto, lo cual se corrobora con las diferencias estadísticamente significativas encontradas en la puntuación de Apgar de los recién nacidos a quienes se les aplicó las espátulas profilácticamente $\mathrm{y}$ aquellos a quienes se les aplicó terapéuticamente.

Las cifras de morbilidad fetal global de $5.73 \%$ referida a huella facial unilateral, hematoma parietal, escoriación en la región frontal y en la región ciliar, edema occipital y parietal y parálisis facial, están por debajo de lo reportado por Beckman y Barreto (4), quienes señalan una morbilidad global de $8 \%$ referida a marcas, equimosis, laceraciones y edema facial; mientras que Ramírez Merchán y Col (20) no describen ninguna morbilidad fetal atribuible al uso de las espátulas.

Consideramos importante señalar que según la nota de egreso, todos los recién nacidos que presentaron las lesiones anteriormente descritas, fueron dados de alta en buenas condiciones en las $24 \mathrm{y}$ 72 horas que siguieron al parto.

\section{Conclusiones}

1. En el quinquenio estudiado, el porcentaje de partos terminados con Espátulas de Velasco varió entre el 
2.24 y 5.78 , notándose una línea ligeramente ascendente con un aumento promedio de $1.1 \%$.

2. La mayor probabilidad de utilización fue en primíparas $(53.16 \%$ ) $y$ en segundo lugar las grandes multíparas $(16.09 \%$ \% $)$.

3. Con respecto a la edad de las parturientas la mayor probabilidad de aplicación $(40.04 \%$ ) correspondió al grupo etario comprendido entre los 15 y los 19 años.

4. La mayor probabilidad de utilización del instrumento en estudio, ocurrió en los embarazos de término $(34.37 \%$ o), siguiendo los postmaduros $(30.54 \%$ \% y finalmente los prematuros $(24.97 \%$ \% $)$.

5. La mayor probabilidad de aplicación (de $48.51 \%$ a $74.02 \%$ ), se observó en aquellas parturientas con un trabajo de parto mayor de once (11) horas.

6. Las espátulas tuvieron un mayor porcentaje de aplicación en los casos de trabajo de parto, con período expulsivo prolongado $(56 \%)$.

7. Todas las aplicaciones estuvieron enmarcadas por debajo del III Plano de Hodge.

8. En la aplicación predominaron las variedades occipito púbicas anteriores $(88.42 \%)$.

9. El $67.04 \%$ de las espátulas aplicadas, tuvieron carácter terapéutico, predominando las indicaciones por expulsivo prolongado y la combinación distocia de rotación, con trabajo de parto prolongado y expulsivo prolongado.
10. Las probabilidades de obtener ur. neonato con puntuación de Apgar igual o mayor de siete (7) son significativamente altas cuando la aplicación de las espátulas se hace profilácticamente.

11. La mayoría de las aplicaciones del instrumento $60.15 \%$ se efectuó, utilizando anestesia pudenda bilateral.

12. En el $90.80 \%$ de los casos no hubo morbilidad materna.

13. La mayor probabilidad de aplicación fue en fetos con peso menores de $2.500 \mathrm{gr}, \mathrm{y}$ en los de peso mayor de $4.000 \mathrm{gr}$.

14. El Apgar de los recién nacidos, a quienes se aplicó Espátulas de Velasco fue igual o mayor de siete (7) puntos en la mayoría de los casos $(88.12 \%)$.

15. En el $94.25 \%$ de los recién nacidos, no hubo morbilidad atribuible al uso del instrumento.

16. No se reportó ningún caso de muerte perinatal atribuible al uso de las espátulas.

17. Los resultados obtenidos en este estudio sobre el manejo de las Espátulas de Velasco como instrumento obstétrico de extracción fetal, son altamente satisfactorios, hemos podido constatar una baja morbilidad materno fetal así como también la ausencia de mortalidad.

\section{Recomendaciones}

Considerando que las Espátulas de Velasco, constituyen un Instrumento de fácil manejo y poca traumaticidad tanto para la madre como para el feto, reco- 
mendamos su uso como instrumento ideal para agilizar la terminación de parto, no sólo con fines terapéuticos, sino también con fines profilácticos.

Tomando en cuenta que en este este estudio, el bajo porcentaje de lesiones maternas encontradas pueden ser más atribuibles a la falta de pericia del operador, que al instrumento en sí, se recomienda el entrenamiento adecuado en el manejo del mismo, a los médicos adscritos a los Servicios Obstétricos, supuestos usuarios del Instrumento.

\section{USE OF VELASCO'S SPATULA AS A FETUS EXTRACTION INSTRUMENT}

\section{Recommendations}

Taking into account that Velasco's Spatulas are easy to handle and have low traumatic effects on both the mother and the fetus, we recommend their use as an ideal instrument to accelerate the completion of the delivery, as well as for therapeutic and prophylactic purposes.

Taking into consideration that the low percentage of injuries to the mother found in this study was mostly the result of the lack of experience of the operator than to the instrument in itself, we recommend that relevant training for its operation be given to the doctors adscribed to the Obstetric Services, who are supposed to be the users of this instrument.

\section{Bibliografía}

1. AGUERO, OSCAR: “Evaluación de fórceps tractores en el eje sin tractor". Rev. Obst. Ginec. Venezuela XXXV,2, 1975. PP. 119-129.

2. ALLER, JUAN: "Obstetricia moderna". Talleres Foto-lito-tipográficos de encua- dernación Franco. Caracas. Venezuela. 1977. P. 463.

3. AURE TULENE, MERCEDES: “Evaluación del fórceps de Hubert-Gilles". Rev. Obst. Ginec. Venezuela XXII, 4, 1962. Pp. 665-668.

4. BECKMANN STEINER, ENRIQUE: BARRETO SHMEEKLING, FRANCISCO JAVIER: “Espátulas “ivs. fórceps". Tra-bajo inédito. Universidad del Rosario, Facultad de Medicina. Bogotá, Colombia. 1975, P. 15.

5. BENSON, RALPH G.: “Diagnóstico y tratamiento ginecoobstétrico". Editorial EI. Manual Moderno, S.A. México. 1979. P. 1.080 .

6. CASTELLANOS, ROSENDO, NAVARRETE, SALVADOR; CABELLO, FRE. DDY; y Colaboradores: “EI fórceps en la maternidad Concepción Palacios 1957. 1959". Rev. Obst. Ginec. Venezuela, XXII, 4, 1972. Pp. 643-653.

7. CARDENAS CONDE, LEOPOLDO; PICO ESPINA, ELEAZAR: "Fórceps de Hawks-Dennen. Nuestra experiencia en cien casos.". Rev. Obst. Ginec. Venezuela XXXVI, 4, 1976,. Pp. 685-693.

8. CHACON VIVAS, G.E.: “EI fórceps en el Hospital Central de San Cristobal durante el quinquenio 1966-1970". Rev. Obst. Ginec. Venezuela XXXVII, 3, 1977. Pp. 327-340.

9. DUBROVSKY, RICARDO: BRUNO AJELLO, FRANCISCO: "Teoría y práctica de fórceps". Editorial Bibliográfica; Argentina, Buenos Aires, 1955 P. 426.

10. FEO CODECIDO, ERNESTO: “Principales métodos instrumentales de extracción fetal". Rev. Obst. Ginec. Venezuela XIX, 2, 1959, Pp. 243-270.

11. GARCIA GAMBOA, MARIO A.: “EI fórceps obstétrico ilustrado su historia $y$ 
evolución". Progresos de Obstetricia y Ginecología. Salvat Editores, S.A. BarceIona, España. XXI, 4, 1978. Pp. 215-222.

12. GARCIA GAMBOA, MARIO A. "Las Espátulas de Velasco". Trabajo inédito Universidad de Puerto Rico. Recinto de Ciencias Médicas. Escuela de Medicina. 1975. P. 27.

13. GREENHILL, J.P.; FRIEDMAN, ENMANUEL A.: "Biological principle and moders practice of obstetrics". W. B. Saunders Company-philadelphia. 1974. P. 837.

14. HUGHES, EDWARD C.: "Terminología en Obstetricia y Ginecología". Salvat Editores, S. A. Barcelona, España. 1975. P. 203.

15. MARTINEZ, PAUL; MARCANO, HECTOR: "Evaluación del fórceps de Laufe en la maternidad Concepción Palacios". Rev. Obst. Ginec. Venezuela, XXXIX, 1, 1979. Pp. 54-56.

16. MONRROY, TULIO; CELLI, B.; FEO CODECIDO, E.; y Colaboradores: “Evaluación de las Espátulas de Thierry". Rev. Obst. Ginec. Venezuela XX, 3, 1960. Pp. 439.476.

17. MULLER, G.; HIGUEREY C., P.; DELGADO DE SULER, A.: y Colaboradores: "Fórceps". Rev. Obst. Ginec. Venezuela. XXXVI, 2, 1976. Pp. 305-311.
18. NAVARRETE ORTA, S.; CASTELLANOS, R.; GARCIA R., M.; y Colaboradores: "Cuatro mil fórceps en la maternidad Concepción Palacios 1960-1962". Rev. Obst. Ginec. Venezuela, XXXIII, 3. 1973. Pp. 443-462.

19. PRITCHARD, JSCKA A.; MACDONALD. PAUL C.: "Wilians Obstetricia". Segunda Edición. Salvat Editores, S.A. Barcelona, Madrid, 1979.

20. RAMIREZ MERCHAN; RAFAEL; VELASCO CH., ALVARO: ARIZA, ALFREDO; y Colaboradores: "Las Espátulas de Velasco Evaluación Clínica". Rev. Colombiana Obst. Ginec. XXII, 2, 1975. Pp. 111-117.

21. URANGA IMAZ, FRANCISCO; URANGA IMAZ, FRANCISCO (h): “Obstetricia práctica". Cuarta Edición. Intermédica Editorial. Buenos Aires, Argentina. 1976. P. 974.

22. UZCATEGUi, OfELIA; ORTEGA, JESUS; PEREZ, ALVARO; GORSKI, JOSEFINA: "Fórceps en el Hospital General Dr. José Gregorio Hernández". Rev. Obst. Ginec. Venezuela, XXXVIII, 2, 1978, Pp. 33-35.

23. VELASCO CHIRIBOGA, ALVARO: "Espátulas para la extracción fetal un nuevo instrumento obstétrico I-descripción general y técnica de aplicación". Rev. Col. Obst. y Ginec. XXIV, 1. 1975. Pp. 17-32. 